TITLE:

\title{
Rhythmic bursting in a cluster of microbeads driven by a continuous-wave laser beam
}

\section{$\operatorname{AUTHOR}(\mathrm{S}):$}

Magome, N; Kitahata, H; Ichikawa, M; Nomura, SIM; Yoshikawa, $\mathrm{K}$

\section{CITATION:}

Magome, N ... [et al]. Rhythmic bursting in a cluster of microbeads driven by a continuous-wave laser beam. Physical Review E 2002, 65(4):

045202.

ISSUE DATE:

2002-04

URL:

http://hdl.handle.net/2433/49857

RIGHT:

Copyright 2002 American Physical Society 
PHYSICAL REVIEW E, VOLUME 65, 045202(R)

\title{
Rhythmic bursting in a cluster of microbeads driven by a continuous-wave laser beam
}

\author{
Nobuyuki Magome, Hiroyuki Kitahata, Masatoshi Ichikawa, Shin-ichiro M. Nomura, and Kenichi Yoshikawa* \\ Department of Physics, Graduate School of Science, Kyoto University and CREST, Kyoto 606-8502, Japan
}

(Received 13 June 2001; published 1 April 2002)

\begin{abstract}
Rhythmic bursting on the order of seconds in a cluster of plastic beads under continuous irradiation of a focused neodymium-doped yttrium aluminum garnet (Nd:YAG) laser beam $(1064 \mathrm{~nm})$ is reported. The oscillatory instability is induced as a result of competition between trapping and scattering forces, where both forces are induced by the focused laser beam. Above a critical power of the laser beam, mode bifurcation from the stationary state into periodic bursting is observed. Our model employing ordinary differential equations reproduces the essential aspects of the experimental results.
\end{abstract}

DOI: 10.1103/PhysRevE.65.045202

PACS number(s): 05.45.Xt, 42.62.-b

Living organisms on the earth maintain their lives under thermodynamically open conditions by energy supplied from the sun. Thus, studies on the production of spatiotemporal structures under light irradiation are of scientific value not only in basic physics but also in the biological sciences. As typical characteristics generated under thermodynamically open conditions, breaking in time-translational symmetry or the appearance of a temporal rhythm would be important in relation to the dynamic aspects of life [1-3]. A rich variety of breaks in the symmetry of time translation in living matter is known, including those of the beating heart, nervous firing, circadian rhythms, cell cycle, etc., where the periodicity is on the order of milliseconds to days [4]. As for nonbiological systems, the self-pulsing of a laser is the representative oscillatory phenomenon generated under far-fromequilibrium conditions, where the periodicity is rather short, being on the order of nanoseconds to microseconds [5-11]. In the present paper, we would like to report a rhythmic phenomenon in the periodic growth and burst of a cluster of submicrometer-sized polystyrene beads in aqueous solution under the irradiation of a focused neodymium-doped yttrium aluminum garnet (Nd:YAG) laser beam.

As has been indicated by Ashkin, an object can be trapped by a focused laser beam, namely, optical tweezers [12]. In general, the force generated with a focused beam is represented as the summation of gradient and scattering terms $[13,14]$

$$
\mathbf{F}=\alpha \boldsymbol{\nabla}\left\langle\mathbf{E}^{2}\right\rangle+\beta \mathbf{E} \times \mathbf{H},
$$

where $\mathbf{E}$ and $\mathbf{H}$ are the electromagnetic field of the focused light, and $\alpha$ and $\beta$ are constants, as a function of the refractive index of the aqueous medium and the trapped object. The first term describes an attractive potential around the focus, where the attractive force decreases with decrease of the convergence angle of the optical cone [see Fig. 1(a)]. In conventional laser-trap experiments, the convergence angle is maximized in order to make the trapping force larger. In contrast, as in the last term in Eq. (1), the scattering force exerted on an object increases with decreases in the conver-

\footnotetext{
*Corresponding author. FAX: +81-75-753-3779. Email address: yoshikaw@scphys.kyoto-u.ac.jp
}

gence angle. It is thus expected that instability in optical trapping would be induced by decreases in the convergence angle [15]. Bearing this effect in mind, we have carried out a laser-trapping experiment with submicrometer-sized plastic beads exhibiting a negative charge. Without laser irradiation, the beads disperse homogeneously in aqueous solution due to their negative charge. A converged laser beam under a standard angle $120^{\circ}$ for the optical cone induces stationary clustering of the beads [16-19]. By changing the convergence angle from $120^{\circ}$ to $80^{\circ}$, a rhythmic change between the growth and the bursting of the cluster is generated, as shown in Fig. 1(b), with the dark region corresponding to the dense clustering of beads trapped on the focus.

We obtained a side view of the cluster by tilting the optical axis (data not shown). The growth and bursting process of the bead cluster has actually been confirmed from such observations of time-dependent changes in the cluster morphology as are schematically depicted in Fig. 1(c). Process (I): Driven by radiation pressure, the beads flow toward the focused region and form a cluster. In processes (II) and (III), the cluster then grows gradually under the trapping potential. In process (IV), when the cluster reaches a critical size, the cluster bursts, and the beads are blown away. Then, growth of the cluster begins again. Such rhythmic change continues under stationary irradiation of the laser.

Figure 2(a) shows a time trace of the cluster size (lefthand side) evaluated from a microscopic image as in Fig. 1(b), together with a frequency spectrum of the autocorrelation, or a power spectrum by fast Fourier transform (FFT) (right-hand side). When the laser power is less than $0.4 \mathrm{~W}$, a small cluster is induced and trapped in a stationary manner. With increase in power, the cluster gradually becomes unstable (e.g., at $0.5 \mathrm{~W}$ ). Above $0.6 \mathrm{~W}$, rhythmic bursting is generated, with almost all of the beads blown away. Further increase in power increases both the frequency and amplitude of oscillation of the cluster size in a gradual manner. Around $1.6 \mathrm{~W}$, the oscillation tends to be irregular. When the power is larger than $1.8 \mathrm{~W}$, no oscillation is observed, i.e., a continuous flow of the beads is generated, with no stationary cluster formation on the focus. Figure 2(b) shows a diagram of cluster size as a function of the laser power, indicating the existence of two different branches. The upper branch is characterized by the clustering of beads around the focus, where the trapped beads stay within the attractive potential 
a)

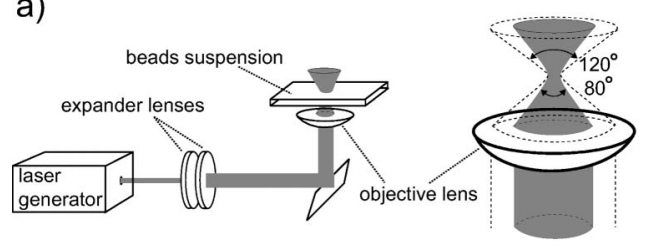

b)

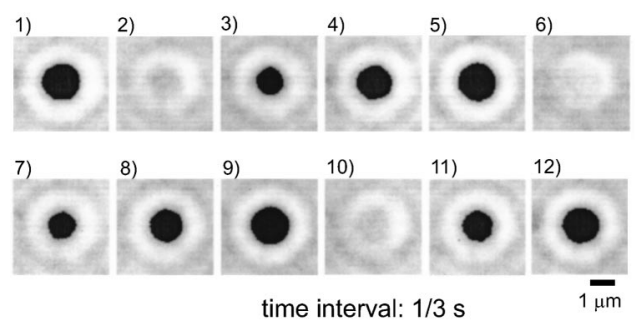

c)

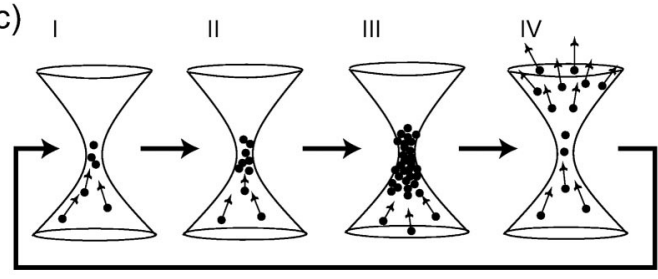

FIG. 1. (a) Schematic representation of the experimental setup. The distance between the expander lenses was adjusted so as to decrease the convergence cone angle, about $80^{\circ}$. (b) Periodic growth and bursting of the bead cluster (black region) observed at 1.0 W. A continuous-wave Nd:YAG laser beam (1064 nm, SL902T, Spectron) was used for the irradiation. The mode of the laser is $\mathrm{TEM}_{00}$, and the profile of the beam has Gaussian distribution as confirmed by a beam profiler (BeamAlyzer, Melles Griot). The laser beam was introduced into an inverted microscope (TE-300, Nikon) through expander lenses and reflecting mirrors, and was converged with a $100 \times$ oil-immersed objective lens. The negatively charged beads of polystyrene latex $(0.20 \mu \mathrm{m}$ in diameter $)$ were purchased from Dow Chemicals. The bead suspension, which contained $0.1 \%$ solids, was situated between glass plates. The thickness of the liquid was approximately $100 \mu \mathrm{m}$. Temperature was $20 \pm 2{ }^{\circ} \mathrm{C}$. (c) Schematic representation of the process of periodic bursting. The laser beam irradiates from below, and the beads flow along the beam axis.

of the focused laser. In the lower branch, the beads do not stay stationary within the attractive potential, but form a continuous flow along the optical cone due to the relatively large effect of optical pressure. Here the density of the beads is higher than in the surrounding suspension, because of a geometrical effect, i.e., the presence of the narrow neck on the optical cone.

Based on the experimental evidence of the existence of the higher and lower branches in the diagram, we discuss the qualitative mechanism by adopting a simple model. For the growth process of the cluster, we take the kinetics as follows:

$$
\dot{n}=k\left(n_{c}-n\right)^{\gamma}-u \text {. }
$$

The first term in Eq. (2) represents the growth rate of the cluster represented by $n$ (the number of beads), $n_{c}$ is the
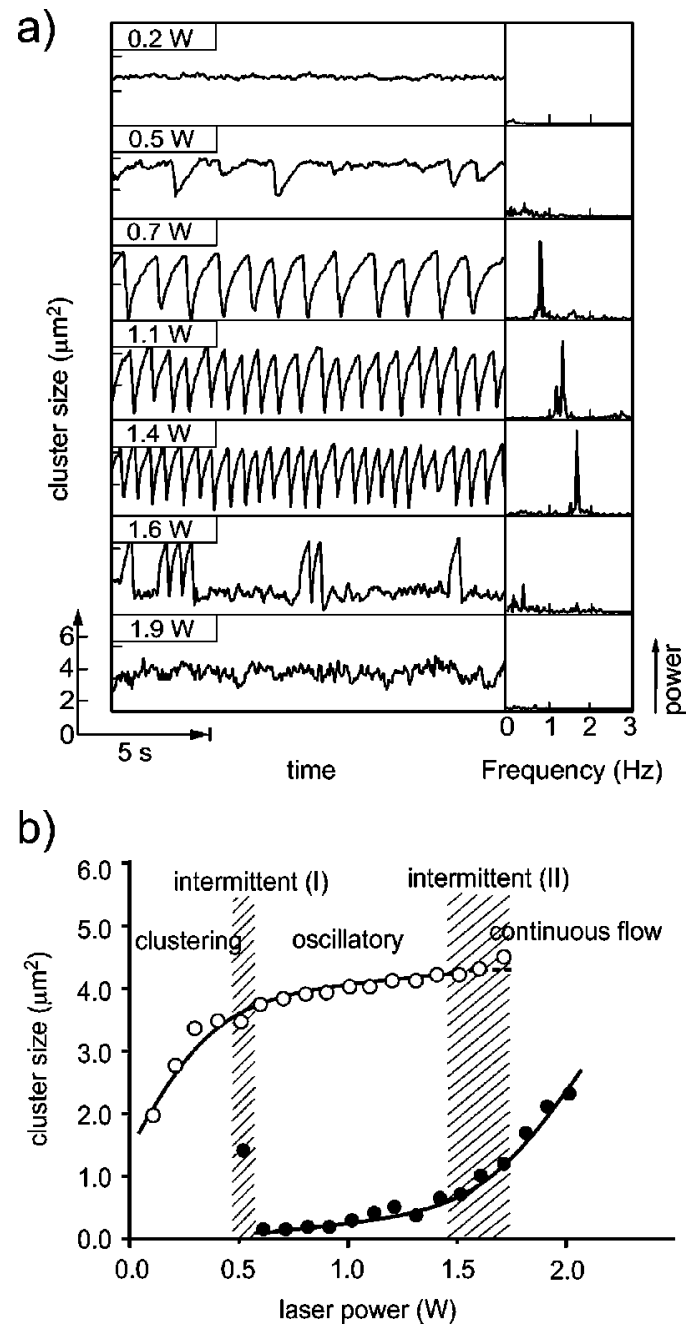

FIG. 2. (a) Time trace of the cluster size with a time interval of $1 / 30 \mathrm{~s}$ (left-hand side) and the power spectrum by FFT analysis (right-hand side). (b) Diagram of cluster size depending on laser power. The cluster sizes were obtained from average values of (1) the basal fluctuation in the "clustering" phase (open circles), (2) the basal fluctuation (open circles) and the lowest values of intermittent decrease (closed circles) in the "intermittent (I)" phase, (3) the tops (open circles) and bottoms (closed circles) of the oscillation in the "oscillatory" phase, (4) the top values of intermittent increase (open circles) and area of dense region (closed circles) generated by continuous bead flow in the "intermittent (II)" phase, and (5) the area of the dense region (closed circles) in the "continuous flow" phase.

upper critical number of beads in a cluster, and $k$ is a function of the attractive force of the laser. As the cluster grows with breaking of spherical symmetry of its shape and also of the optical field, the actual growth rate is rather complicated. In order to describe the essence of the oscillation, we introduce a simple function to exhibit the growth rate with a single parameter $\gamma$. It is expected that $\gamma=1$ and $2 / 3$ when the cluster grows in a cylindrical shape with the same diameter and in a genuine spherical shape, respectively, under the condition that beads colliding with the cluster stick to it in an efficient manner. In this calculation, it is supposed that $\gamma$ $=0.5$. The second term $u$ corresponds to the rate of escape 
from the cluster. For the time-dependent change of the flow rate, $\dot{u}$, we adopt a relationship as follows:

$$
\dot{u}=n-(u-a)^{\eta}+c u-b,
$$

where $a, b$, and $c$ are constants. The first term represents the linear increase of flow rate with the increase of cluster size. The other terms are given in order to take into account of the characteristic features shown in Fig. 2(b). For the nonlinear term, we simply take $\eta=3$. In other words, we consider a model system of coupled differential equations, Eqs. (2) and (3), where cubic nonlinearity is introduced into Eq. (3), so as to interpret the presence of the two branches.

Figure 3(a) shows the time-dependent changes of the number of beads in a cluster and the power spectrum by FFT analysis, as calculated from Eqs. (2) and (3). In this numerical calculation, we have changed $k$ as the representative parameter of the laser power. The cluster stays stationary below a lower critical value of $k$. When $k$ is between 0.33 and 0.86 , the cluster begins to exhibit the periodic oscillation of growth and bursting. Above the upper critical value, 0.9, no cluster oscillation is exhibited. Figure 3(b) shows the phase diagram as a function of $k$. Behavior of the clustering phenomenon as indicated in Figs. 3(a) and 3(b) corresponds well with the experimental results, despite the simple and rough modeling. From the above results and discussion, the rhythmic behavior is explained as follows. In the growth phase, the cluster size gradually increases [corresponding to Fig. $1(\mathrm{c})$, processes I-III]. Accompanying this growth, the effective light pressure acting on the cluster increases, and the vertical position of the center of mass of the cluster tends to rise. This makes the cluster less stable with increase of cluster size. As a result, the cluster bursts due to the intrinsic repulsive nature between the beads [Fig. 1(c), process IV]. Then the cycles of growth and bursting are repeated.

In conclusion, we have shown the appearance of oscillatory instability in a cluster of beads under continuous laser irradiation by choosing an appropriate angle of the optical cone. This oscillation is caused by the competition between the trapping and scattering forces exerted by the focused laser, for a system of negatively charged beads that repel each other.
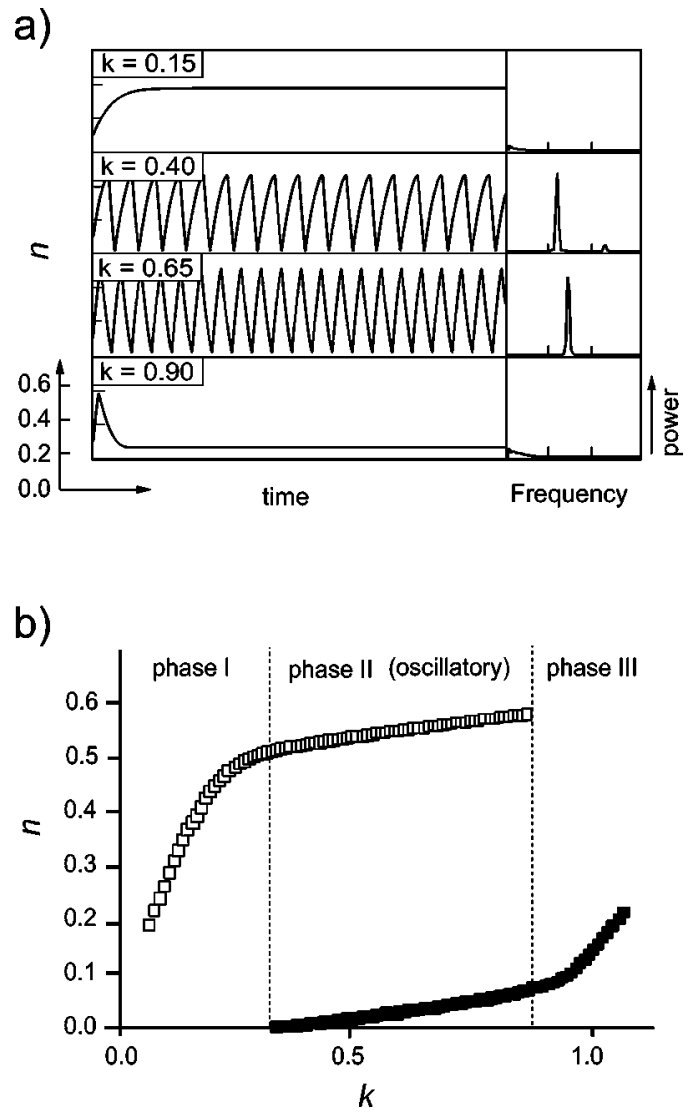

FIG. 3. (a) Numerical simulation of the rhythmic bursting. Time trace of the number of beads $n$ (left-hand side), and the power spectrum (right-hand side) are shown. The fourth Runge-Kutta method was used, and the set of parameters $a=1.0, b=1.0, c$ $=0.7, n_{c}=3.0, \gamma=0.5$, and $\eta=3.0$ was chosen for the simulation. (b) Diagram of $n$ depending on $k$. The values of $n$ were obtained from (1) the values at the steady state (open squares) in "phase I," (2) the top values (open squares) and bottom values (closed squares) of the oscillation in "phase II," and (3) the values at the steady state (closed squares) in "phase III." All units are arbitrary.

The authors would like to thank Dr. T. Akitaya, Dr. H. Mayama, and T. Harada for their helpful discussion and comments. The present study was supported in part by the Yamada Science Foundation.
[1] Chemical Waves and Patterns, edited by R. Kapral and K. Showalter (Kluwer Academic Publishers, Dordrecht, 1995).

[2] G. Nicolis and I. Prigogine, Self-Organization in Nonequilibrium Systems (Wiley, New York, 1977).

[3] H. Haken, Synergetics (Springer-Verlag, Berlin, 1978).

[4] A.T. Winfree, The Geometry of Biological Time (SpringerVerlag, Berlin, 1980).

[5] P. Meystre and M. Sargent, Elements of Quantum Optics (Springer-Verlag, Berlin, 1990).

[6] H. Haken, Phys. Lett. 53A, 77 (1975).

[7] F. Prati, E.M. Pessina, G.J. de Valcárcel, and E. Roldán, Opt. Commun. 185, 153 (2000).
[8] P. Kafalas, J.I. Masters, and E.M.E. Murray, J. Appl. Phys. 35, 2349 (1964).

[9] O.R. Wood and S.E. Schwarz, Appl. Phys. Lett. 11, 88 (1967).

[10] M. Lando, Y. Shimony, Y. Noter, R.M.J. Benmair, and A. Yogev, Appl. Opt. 39, 1962 (2000).

[11] W. Zendzian, J.K. Jabczynski, and Z. Mierczyk, OptoElectron. Rev. 9, 75 (2001).

[12] A. Ashkin, Phys. Rev. Lett. 24, 156 (1970).

[13] Y.R. Shen, The Principles of Nonlinear Optics (Wiley, New York, 1984).

[14] K. Svoboda and S.M. Block, Annu. Rev. Biophys. Biomol. Struct. 23, 247 (1994). 
[15] G.J. Sonec and W. Wang, Rev. Laser Eng. 24, 1139 (1996).

[16] J. Won, T. Inaba, H. Matsuhara, H. Fujiwara, K. Sasaki, S. Miyawaki, and S. Sato, Appl. Phys. Lett. 75, 1506 (1999).

[17] T.A. Smith, J. Hotta, K. Sasaki, H. Masuhara, and Y. Ito, J. Phys. Chem. B 103, 1660 (1999).
[18] J. Hofkens, J. Hotta, K. Sasaki, H. Matsuhara, and K. Iwai, Langmuir 13, 414 (1997).

[19] J. Hotta, K. Sasaki, and H. Matsuhara, J. Am. Chem. Soc. 118, 11968 (1996). 